\title{
Robust Integer Programming
}

\author{
Shmuel Onn \\ Technion - Israel Institute of Technology, Haifa, Israel \\ onn@ie.technion.ac.il
}

\begin{abstract}
We provide a complexity classification of four variants of robust integer programming when the underlying Graver basis is given. We discuss applications to robust multicommodity flows and multidimensional transportation, and describe an effective parametrization of robust integer programming.
\end{abstract}

\section{Introduction}

Robust discrete optimization involves making optimal decisions under uncertainty in the problem data, see [4] for a detailed development of this framework. In this note we study robust integer programming problems where the uncertainty occurs in the cost of the feasible points. More precisely, we consider a set of feasible integer points which satisfy a system of linear inequalities in standard form given by

$$
X:=\left\{x \in \mathbb{Z}^{n}, A x=b, l \leq x \leq u\right\},
$$

where $A$ is an integer $m \times n$ matrix, $b \in \mathbb{Z}^{m}$, and $l, u \in \mathbb{Z}^{n}$. The uncertain cost to be minimized belongs to a finite nonempty set $C \subset \mathbb{Z}^{n}$ of potential cost vectors. The worst-case cost of point $x \in X$ is $\max \{c x: c \in C\}$. The robust integer programming problem is then to find $x \in X$ attaining minimum worst-case cost, that is, to solve

$$
\min _{x \in X} \max _{c \in C} c x
$$

When $C$ is a singleton, the problem reduces to standard linear integer programming, and hence is no easier than the latter, which is NP-hard. We therefore make some reasonable assumptions on $C$ and $X$ and study the complexity of the problem under these assumptions. First, we assume that $C$ is either an explicit list $C=\left\{c^{1}, \ldots, c^{k}\right\}$ of given cost vectors, or a box $C=\left\{c \in \mathbb{Z}^{n}: d \leq c \leq e\right\}$ for some $d, e \in \mathbb{Z}^{n}$, so that the cost of each decision variable lies in a given interval. Note that a box $C$ has exponentially many elements and cannot be explicitly listed efficiently. Second, we assume that the so-called Graver basis $\mathcal{G}(A)$ of the matrix $A$ defining $X$ is given; 
this object, defined in Section 2, plays a central role in a recent theory of integer programming developed in [5] and the references therein. As illustrated in Section 3, the Graver basis can be computed in polynomial time from $A$ for a variety of applications including multicommodity flows and multidimensional transportation. Moreover, there is a parametrization of all integer programs [2] under which the Graver basis can be computed in polynomial time for each fixed parameter value.

The standard interpretation of robust discrete optimization is that the "decision maker" first chooses a point from $X$ and that "nature" then chooses a cost from $C$. However, the problem can more symmetrically be regarded as a two party game where the $X$ player must pay $c x$ to the $C$ player. It is then also natural to consider the "dual" problem where the $C$ player makes its choice first, that is, the problem

$$
\max _{c \in C} \min _{x \in X} c x .
$$

We show the following complexity classification of robust integer programming.

Theorem 1.1 The following complexity table holds for the robust integer programming problems with the Graver basis of the matrix of $X$ given as part of the input:

\begin{tabular}{|c|cc|}
\hline & list $C$ & box $C$ \\
\hline $\min _{X} \max _{C}$ & NP-hard & polynomial time \\
$\max _{C} \min _{X}$ & polynomial time & NP-hard \\
\hline
\end{tabular}

We remark that when costs are replaced by profits, the corresponding problems reduce to the corresponding problems with $C$ replaced by $-C:=\{-c: c \in C\}$ via the following relations, resulting in the same complexities as in Theorem 1.1:

$$
\max _{x \in X} \min _{c \in C} c x=-\min _{x \in X} \max _{c \in-C} c x, \quad \min _{c \in C} \max _{x \in X} c x=-\max _{c \in-C} \min _{x \in X} c x .
$$

In Section 2 we define the Graver basis and prove Theorem 1.1. In Section 3 we discuss applications to robust multicommodity flows and robust multidimensional transportation, and describe the parametrization of robust integer programming.

\section{Proof}

We begin by defining the Graver basis. Introduce a partial order $\sqsubseteq$ on $\mathbb{R}^{n}$ by $x \sqsubseteq y$ if $x_{i} y_{i} \geq 0$ and $\left|x_{i}\right| \leq\left|y_{i}\right|$ for $i=1, \ldots, n$. The Graver basis of the integer $m \times n$ matrix $A$ is the finite set $\mathcal{G}(A) \subset \mathbb{Z}^{n}$ of $\sqsubseteq$-minimal elements in $\left\{x \in \mathbb{Z}^{n}: A x=0, x \neq 0\right\}$.

Proof of Theorem 1.1. We prove the four entries of the complexity table one by one.

Entry (1,1): Consider the NP-complete problem of deciding, given $a \in \mathbb{Z}_{+}^{n}$, if there is a subset $I \subseteq[n]:=\{1, \ldots, n\}$ such that $\sum_{i \in I} a_{i}=\sum_{i \notin I} a_{i}$. Let $a_{0}:=\sum_{i=1}^{n} a_{i}$ and define $c^{1}, c^{2} \in \mathbb{Z}^{n+1}$ by $c^{1}:=(0, a)$ and $c^{2}:=\left(a_{0},-a\right)$. Next, define the set

$$
X:=\left\{x \in \mathbb{Z}^{n+1}: \mathbf{0} x=0, \quad 1 \leq x_{0} \leq 1, \quad 0 \leq x_{i} \leq 1, \quad i=1, \ldots, n\right\}
$$


it is not hard to verify that the Graver basis of the matrix $\mathbf{0}$ defining $X$ is given by $\mathcal{G}(\mathbf{0})= \pm\left\{\mathbf{1}_{i}: 0 \leq i \leq n\right\}$ with $\mathbf{1}_{i}$ the $i$ th unit vector. Now, there is a bijection between $x \in X$ and $I \subseteq[n]$ with $I(x):=\left\{i: 1 \leq i \leq n, x_{i}=1\right\}$. For any $x \in X$,

$$
\max \left\{c^{1} x, c^{2} x\right\}=\max \left\{\sum_{i \in I(x)} a_{i}, a_{0}-\sum_{i \in I(x)} a_{i}\right\}=\max \left\{\sum_{i \in I(x)} a_{i}, \sum_{i \notin I(x)} a_{i}\right\} \geq \frac{a_{0}}{2}
$$

with equality if and only $\sum_{i \in I(x)} a_{i}=\sum_{i \notin I(x)} a_{i}$. So $\min _{x \in X} \max _{c \in C} c x=\frac{a_{0}}{2}$ if and only if there is an $I$ with $\sum_{i \in I} a_{i}=\sum_{i \notin I(x)} a_{i}$, and solution of the robust integer programming problem will enable solution of the given NP-complete problem.

Entry (1,2): For of each feasible point $x \in X$ define $f(x):=\max _{c \in C} c x$. Then

$$
f(x):=\max \left\{\sum_{i=1}^{n} c_{i} x_{i}: d_{i} \leq c_{i} \leq e_{i}\right\}=\sum_{i=1}^{n} \max \left\{d_{i} x_{i}, e_{i} x_{i}\right\}=\sum_{i=1}^{n} f_{i}\left(x_{i}\right),
$$

where $f_{i}\left(x_{i}\right):=\max \left\{d_{i} x_{i}, e_{i} x_{i}\right\}$. Since $f_{i}\left(x_{i}\right)$ is the maximum of two univariate convex functions, it is also univariate convex. Therefore $f(x)=\sum_{i=1}^{n} f_{i}\left(x_{i}\right)$ is separable convex. So the robust integer programming problem is the integer program

$$
\min _{x \in X} \max _{c \in C} c x=\min \left\{f(x): x \in \mathbb{Z}^{n}, A x=b, l \leq x \leq u\right\}
$$

of minimizing a separable convex function $f$ over integer points satisfying lower and upper bounds and a system of equations with defining matrix whose Graver basis $\mathcal{G}(A)$ is given, which can be solved in polynomial time, see [5, Theorem 3.12] or [3].

Entry (2,1): For each cost vector $c \in C$ in the given list, consider the program

$$
g(c):=\min \left\{c x: x \in \mathbb{Z}^{n}, A x=b, l \leq x \leq u\right\}
$$

its objective function $c x$ is linear hence separable convex, and therefore, given $\mathcal{G}(A)$, can be solved in polynomial time by [5. Theorem 3.12] again; then that $c \in C$ which attains maximum value $g(c)$ solves the given robust integer programming problem.

Entry (2,2): Consider again the NP-complete problem of deciding, given $a \in \mathbb{Z}_{+}^{n}$, if there is a subset $I \subseteq[n]$ such that $\sum_{i \in I} a_{i}=\sum_{i \notin I} a_{i}$. Let again $a_{0}:=\sum_{i=1}^{n} a_{i}$. Let

$$
C:=\left\{c \in \mathbb{Z}^{n+2}: 1 \leq c_{0} \leq 1, \quad 0 \leq c_{i} \leq 1, \quad i=1, \ldots, n, \quad 0 \leq c_{n+1} \leq 0\right\}
$$

Next let $m:=n+1$ and define an $m \times(n+2)$ matrix $A$ and vector $b \in \mathbb{Z}^{m}$ by

$$
A:=\left(\begin{array}{rr}
I_{n+1} & a_{0} \\
-2 a
\end{array}\right), \quad b:=\left(\begin{array}{r}
0 \\
-a
\end{array}\right)
$$

and let

$$
X:=\left\{x \in \mathbb{Z}^{n+2}: A x=b, \quad-\left|a_{0}\right| \leq x_{i} \leq\left|a_{0}\right|, \quad i=0, \ldots, n, \quad 0 \leq x_{n+1} \leq 1\right\} .
$$


It is not hard to verify that the Graver basis of $A$ is given by $\mathcal{G}(A)= \pm\left\{\left(-a_{0}, 2 a, 1\right)\right\}$.

Now, note that the value of $x_{n+1}$ determines the value of the other $x_{i}$ via the system $A x=b$ so that $X=\left\{(0,-a, 0),\left(-a_{0}, a, 1\right)\right\}$. Next, there is a bijection between $c \in C$ and $I \subseteq[n]$ with $I(c):=\left\{i: 1 \leq i \leq n, c_{i}=1\right\}$. For each $c \in C$,

$$
\min _{x \in X} c x=\min \left\{-\sum_{i \in I(c)} a_{i},-a_{0}+\sum_{i \in I(c)} a_{i}\right\}=\min \left\{-\sum_{i \in I(c)} a_{i},-\sum_{i \notin I(c)} a_{i}\right\} \leq-\frac{a_{0}}{2}
$$

with equality if and only $\sum_{i \in I(c)} a_{i}=\sum_{i \notin I(c)} a_{i}$. So $\max _{c \in C} \min _{x \in X} c x=-\frac{a_{0}}{2}$ if and only if there is an $I$ with $\sum_{i \in I} a_{i}=\sum_{i \notin I} a_{i}$, and solution of the robust integer programming problem will enable solution of the given NP-complete problem.

\section{Applications}

An $(r, s) \times t$ bimatrix is a matrix $A$ consisting of two blocks $A_{1}, A_{2}$, with $A_{1}$ its $r \times t$ submatrix consisting of the first $r$ rows and $A_{2}$ its $s \times t$ submatrix consisting of the last $s$ rows. The $n$-fold product of $A$ is the following $(r+n s) \times n t$ matrix,

$$
A^{(n)} \quad:=\left(\begin{array}{cccc}
A_{1} & A_{1} & \cdots & A_{1} \\
A_{2} & 0 & \cdots & 0 \\
0 & A_{2} & \cdots & 0 \\
\vdots & \vdots & \ddots & \vdots \\
0 & 0 & \cdots & A_{2}
\end{array}\right) .
$$

For each fixed bimatrix $A$, the Graver basis $\mathcal{G}\left(A^{(n)}\right)$ of the $n$-fold product of $A$ can be computed in time which is polynomial in $n$, see [5, Theorem 4.4] or [1]. This has a variety of applications including multicommodity flows, multidimensional transportation, and more generally any integer program via a suitable parametrization. We now discuss consequences of this to the robust counterparts of these applications.

The (integer) multicommodity flow problem is as follows. There are $l$ types of discrete commodities, $m$ suppliers, and $n$ consumers. Supplier $i$ has supply $s_{i}^{k}$ in commodity $k$. Consumer $j$ has demand $d_{j}^{k}$ in commodity $k$. Channel $(i, j)$ has capacity $u_{i, j}$ which is an upper bound on the total flow of all commodities on that channel. There is a cost $c_{i, j}^{k}$ per unit flow of commodity $k$ on channel $(i, j)$. The non-robust problem is to find a multicommodity flow consisting of $x_{i, j}^{k}$ units of flow of commodity $k$ on channel $(i, j)$ for all $i, j, k$, satisfying the supply, demand, and capacity constraints, and attaining minimum cost $\sum_{i, j, k} c_{i, j}^{k} x_{i, j}^{k}$, that is, solve

$$
\min \left\{c x: x=\left(x_{i, j}^{k}\right) \in \mathbb{Z}_{+}^{l \times m \times n}, \sum_{j} x_{i, j}^{k}=s_{i}^{k}, \quad \sum_{i} x_{i, j}^{k}=d_{j}^{k}, \quad \sum_{k=1}^{l} x_{i, j}^{k} \leq u_{i, j}\right\}
$$


The problem is NP-hard already for $l=2$ commodities or $m=3$ suppliers. However, it is natural to have relatively small numbers of commodities and suppliers but very large number of consumers, and we have the following consequence of our theorem.

Corollary 3.1 For fixed l commodities and $m$ suppliers, the robust multicommodity flow problem $\min _{X} \max _{C}$ with box $C$ or $\max _{C} \min _{X}$ with list $C$ is polytime solvable.

Proof. Introduce a slack commodity 0 with new variables $x_{i, j}^{0}:=u_{i, j}-\sum_{k=1}^{l} x_{i, j}^{k}$ representing the slack flow on each channel $(i, j)$, with cost $c_{i, j}^{0}:=0$, and suitable slack supplies $s_{i}^{0}:=\sum_{j} u_{i, j}-\sum_{k=1}^{l} s_{i}^{k}$ and slack demands $d_{j}^{0}:=\sum_{i} u_{i, j}-\sum_{k=1}^{l} d_{j}^{k}$. Let $\hat{C}:=\{(0, c): c \in C\}$ consist of the costs augmented with the 0 slack costs, and

$\hat{X}:=\left\{x \in \mathbb{Z}^{(l+1) \times m \times n}, \sum_{j} x_{i, j}^{k}=s_{i}^{k}, \sum_{i} x_{i, j}^{k}=d_{j}^{k}, \sum_{k=0}^{l} x_{i, j}^{k}=u_{i, j}, 0 \leq x_{i, j}^{k} \leq u_{i, j}\right\}$

It can be shown that the matrix defining the equations of $\hat{X}$ is an $n$-fold product of a fixed bimatrix, hence its Graver basis can be computed in polynomial time, see [5, Chapter 4]. The corollary now follows from Theorem 1.1 applied to $\hat{X}$ and $\hat{C}$.

Next we consider the (integer) three-dimensional transportation problem,

$$
\min \left\{c x: x \in \mathbb{Z}_{+}^{l \times m \times n}: \sum_{i} x_{i, j, k}=u_{j, k}, \sum_{j} x_{i, j, k}=v_{i, k}, \sum_{k} x_{i, j, k}=w_{i, j}\right\}
$$

It is NP-hard even for $l=3$, see [2]. But we have the following robustness statement.

Corollary 3.2 For every fixed $l$ and $m$, the robust three-dimensional transportation problem $\min _{X} \max _{C}$ with box $C$ or $\max _{C} \min _{X}$ with list $C$ is polytime solvable.

Proof. Note that each variable obeys the bounds $0 \leq x_{i, j, k} \leq \min \left\{u_{j, k}, v_{i, k}, w_{i, j}\right\}$. It can be shown again that the matrix defining the equations of $X$ is an $n$-fold product of a fixed bimatrix, hence its Graver basis can again be computed in polynomial time, see [5, Chapter 5]. The corollary then follows again from Theorem 1.1.

It was shown in [2] that every bounded integer program can be isomorphically represented in polynomial time as some $3 \times m \times n$ transportation problems with $l=3$ and some $m$ and $n$. Regarding $m$ as a parameter, Corollary 3.2 implies that we can do robust integer programming in polynomial time for the class of $3 \times m \times n$ problems with varying $n$. Since every integer program belongs to one of these classes for some $m$, this provides an effective parametrization of robust integer programming. 


\section{References}

[1] De Loera, J., Hemmecke, R., Onn, S., Weismantel, R.: N-fold integer programming. Discrete Optimization 5:231-241 (2008)

[2] De Loera, J., Onn, S.: All linear and integer programs are slim 3-way transportation programs. SIAM Journal on Optimization 17:806-821 (2006)

[3] Hemmecke, R., Onn, S., Weismantel, R.: A polynomial oracle-time algorithm for convex integer minimization. Mathematical Programming 126:97-117 (2011)

[4] Kouvelis, P., Yu, G.: Robust Discrete Optimiation and its Applications. Kluwer (1997)

[5] Onn, S.: Nonlinear Discrete Optimization. Zurich Lectures in Advanced Mathematics, European Mathematical Society (2010), available online at: http://ie.technion.ac.il/ onn/Book/NDO.pdf 